\title{
Synchronous melanomas arising within nevus spilus ${ }^{*}$
}

\author{
Maria Helena Toda Sanches de Brito ${ }^{1}$ \\ Cândida Margarida Branco Martins Fernandes ${ }^{3}$ \\ Maria Joaninha Madalena de Palma Mendonça da Costa Rosa ${ }^{4}$
}

\author{
Cecília Silva Nunes de Moura Dionísioº \\ Joana Cintia Monteiro Ferreira ${ }^{4}$ \\ Maria Manuela Antunes Pecegueiro da Silva Garcia²
}

DOI: http:/ /dx.doi.org/10.1590/abd1806-4841.20175230

\begin{abstract}
Nevus spilus is a melanocytic cutaneous lesion consisting of a light brown background macule with numerous superimposed darker maculopapular speckles. Melanoma arising from a nevus spilus is rare, with less than 40 cases reported to date. The absolute risk for malignant transformation is not well defined, lacking a standardized management approach. We report a new case of melanoma arising from nevus spilus, with the additional peculiarity of multifocality. We offer our recommendations for the management of the condition.
\end{abstract}

Keywords: Melanoma; Nevi and melanomas; Nevus

\section{INTRODUCTION}

Nevus spilus (NS) is a relatively uncommon skin lesion found in $0.2 \%-2.3 \%$ of the general population. It is characterized by darkly pigmented macules and papules with background hyperpigmentation..$^{1-5}$ Despite the reports of melanoma arising within NS, the malignant potential of this entity remains unclear. ${ }^{1}$ We report the case of a patient who presented with synchronous melanomas arising from NS.

\section{CASE REPORT}

An 83-year-old Caucasian male was referred to our department for evaluation of a recent change in a pre-existing NS on his right upper extremity. The patient reported that the lesion was present since birth and had showed no significant changes until a year before, when he noticed three darker areas showing continuous growth. He had skin phototype III and a history of significant intermittent sun exposure during childhood and early adulthood. He reported no personal or family history of melanoma or dysplastic nevi.

Examination revealed a $27 \mathrm{~cm} \times 10 \mathrm{~cm}$ light tan patch with speckled lentiginous pigmentation involving the external aspect of his right arm and forearm (Figure 1). Three separate areas were observed within this larger lesion with a varied darker pigmentation and irregular borders: two maculopapules measuring $23 \times 23 \mathrm{~mm}$ (lesion A) and $15 \times 22 \mathrm{~mm}$ (lesion B), and one macule measuring $20 \times 23 \mathrm{~mm}$ (lesion C) (Figure 2). In addition, a $15 \times 13 \mathrm{~mm}$ irregularly pigmented macule was observed on his upper back (lesion D) (Figure 2). Dermatoscopic examination of the four suspicious lesions was highly suggestive of malignant melanoma (Figure 3). No palpable lymphadenopathy was present.

A punch biopsy was performed in lesion A, and histopathological analysis revealed superficial spreading melanoma with a Breslow thickness of $1.15 \mathrm{~mm}$. The patient subsequently underwent wide excisions of each suspicious lesion with a 1-cm margin and sentinel lymph node biopsy. On final pathology, lesions A and B were superficial spreading melanomas, with Breslow thicknesses $2.51 \mathrm{~mm}$ and $1.18 \mathrm{~mm}$, respectively; lesions $\mathrm{C}$ and $\mathrm{D}$ were in situ malignant melanoma (Figure 4). In all cases, ulceration and satellite nodules were absent, lymphovascular or perineural invasion were not seen, and the margins were negative. The intervening epidermis between the neoplastic foci was consistent with a background NS lesion (Figure 5). The sentinel lymph node biopsy, as well as computed tomographic scan, showed no evidence of metastatic disease. It was decided not to pursue additional therapy, and the residual NS lesion is being followed up closely by the Department of Dermatology.

Received on 06.10.2015.

Approved by the Advisory Board and accepted for publication on 23.12.2015.

Work performed at the Department of Dermatology, Instituto Português de Oncologia de Lisboa - Lisboa, Portugal.

Financial Support: None.

Conflict of Interest: None.

Department of Dermatology, Santarém Hospital - Santarém, Portugal.

Department of Dermatology, Instituto Português de Oncologia de Lisboa - Lisboa, Portugal.

Department of Dermatology, Hospital de Santo António dos Capuchos, Centro Hospitalar de Lisboa Central - Lisboa, Portugal.

Department of Pathology, Instituto Português de Oncologia de Lisboa - Lisboa, Portugal.

(C2017 by Anais Brasileiros de Dermatologia 


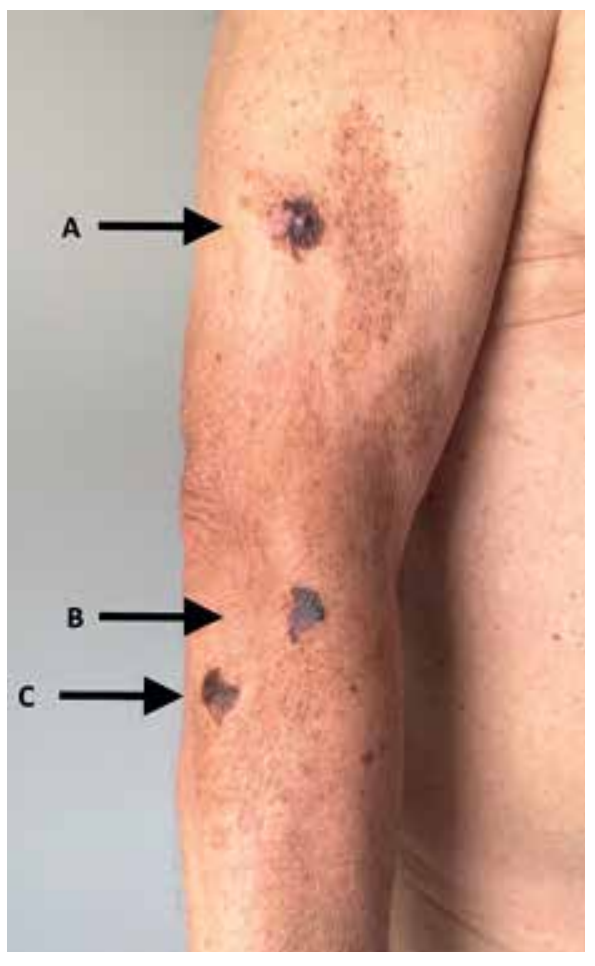

Figure 1:

Clinical picture showing the complete feature of NS, involving the external aspect of the right arm and forearm of the patient. Three foci of newly formed, irregular dark areas can be seen (lesions $\mathrm{A}, \mathrm{B}$, and $\mathrm{C}$ )
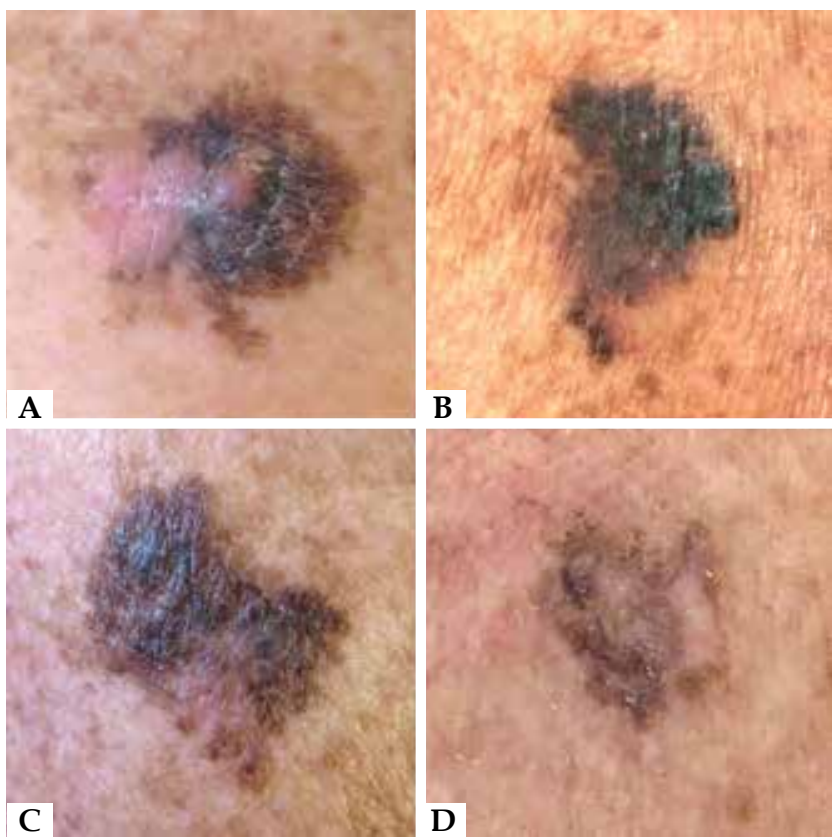

Figure 2: Closer view of the lesions A, B, C and the irregular patch on the upper back of the patient (lesion D)

\section{DISCUSSION}

NS, also known as nevus on nevus, spotty nevus, spot on spot, or speckled lentiginous nevus, is a clinicopathologic entity characterized by a light tan-to-brown background macule speckled with varying numbers of smaller, darker macules or papules. ${ }^{1-6} \mathrm{NS}$ lesions occur fairly equally in men and women and within different races; they may be present at birth or have its onset in childhood, or even after the age of 20 years. ${ }^{3,4,6}$
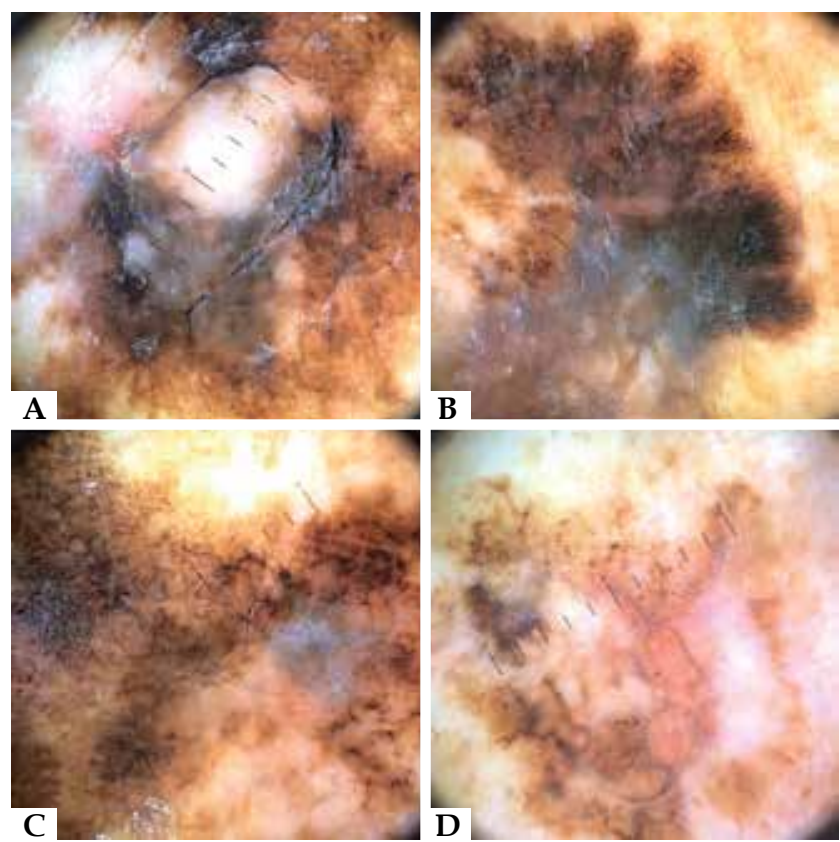

Figure 3: Dermoscopy of the suspected lesions revealed blue-grey veils, structureless areas, and irregular pigment network with black dots and globules
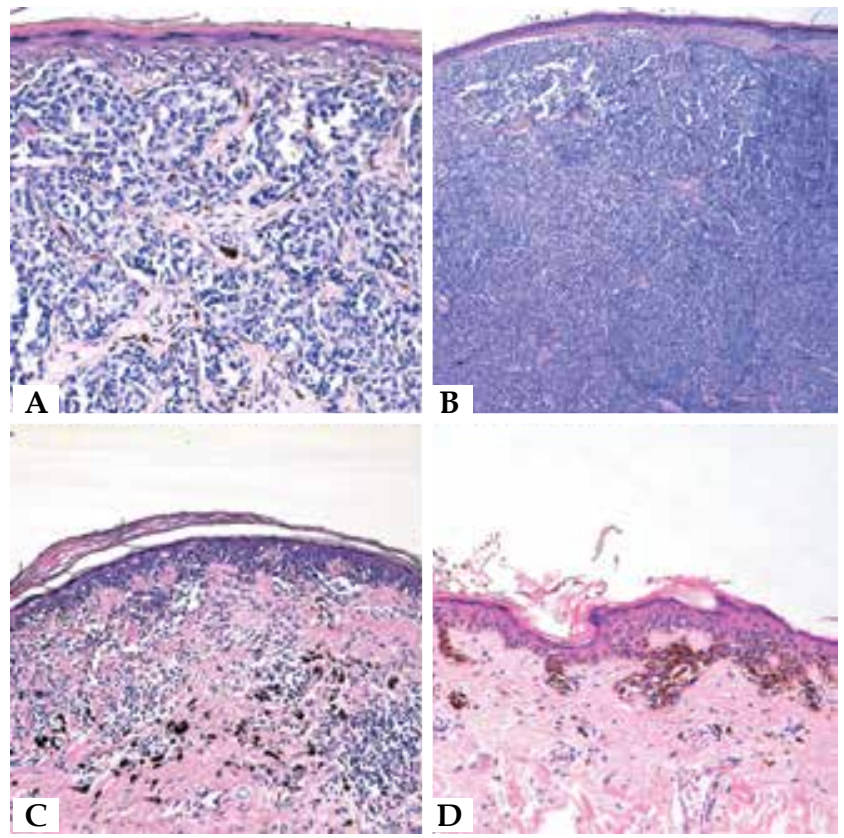

FIGURE 4: Histopathologic examination revealed that lesions A and $B$ were invasive superficial spreading melanomas, forming multiple irregular nests and sheets in the dermis (A_Hematoxylin \& eosin, x100, B_Hematoxylin \& eosin, x40). Histology of lesions C and D showed the characteristics of in situ malignant melanoma (Hematoxylin \& eosin x100), with marked proliferation of atypical melanocytes along the dermal-epidermal junction and superficial inflammatory infiltration of the dermis

Clinically, NS generally consists of a single non-hairy lesion, with a medium size of $4.3 \mathrm{~cm}( \pm 3.5 \mathrm{~cm}){ }^{4,6}$ Spotting within the lesion is more often macular than papular, sized 1-3 mm, and develop during a period of months to years. ${ }^{2,6}$ NS can be divided into macu- 


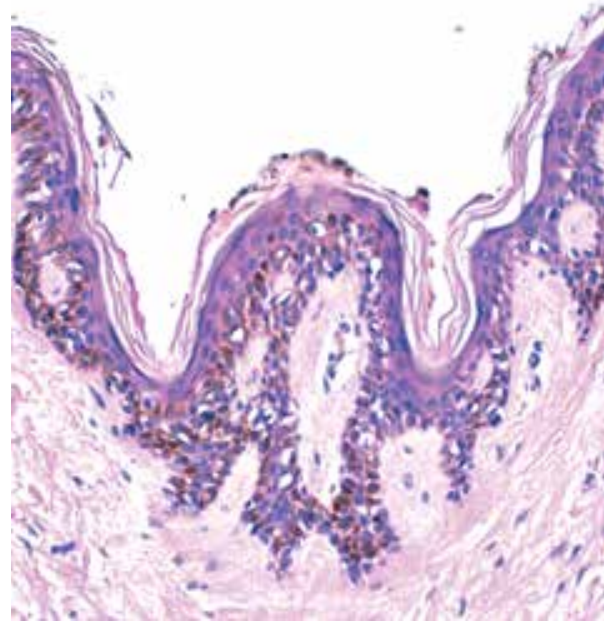

Figure 5:

His topathology of the nevus spilus showing mild acanthosis, elongation of rete ridges, and lentiginous proliferation of basal melanocytes at the dermoepidermal junction with hyperpigmentation of basal keratinocytes (Hematoxylin \& $\operatorname{eosin} \times 200$ )

lar and papular variants, and further into three different size groups: small $(<1.5 \mathrm{~cm})$; medium $(1.5-19.9 \mathrm{~cm})$; and giant $(>20 \mathrm{~cm}) \cdot{ }^{2-4,6}$ Less frequent segmental/zosteriform NS has also been described. ${ }^{2-4,6}$ Lesions can be found anywhere on the body, but involve especially the trunk and extremities. ${ }^{2-4,6}$ On histology, the background pigmented lesion of NS usually corresponds to a lentigo simplex or a café au lait macule, while the dark speckles show features of a variety of nevi: junctional, compound, intradermal, dysplastic, Spitz, blue, neurotized nevus, or ink spot lentigo. ${ }^{2-4,6}$

For a long time, NS was believed to be a harmless pigmented lesion. However, in 1957 Perkinson reported a melanoma appearing on NS for the first time. ${ }^{2,4,6,7}$ Since then, less than 40 cases of melanoma developing within this entity have been published, sometimes with a fatal outcome. ${ }^{1-10}$ Reviewing the published literature, most cases of malignant transformation were found in Caucasian or black patients, more frequently on the trunk (64\%) than the limbs (36\%), with a female-male ratio of 56:44 and a mean age of 49 years. ${ }^{1,2,6} \mathrm{Mel}-$ anoma on NS was usually one neoplasm, but multiple melanomas were observed within the same NS in three previous cases. ${ }^{5,6,8}$ The melanoma most found was superficial spreading melanoma $(68 \%)$, followed by nodular (16\%) and in situ melanoma $(16 \%)^{2,6}$. In $60 \%$ of cases, melanoma developed on small or medium-sized NS, $24 \%$ on zosteriform NS, and $16 \%$ on giant NS. ${ }^{2,6}$ In $52 \%$ of cases, NS was present at birth; in 33\%, it was acquired during infancy; and in $14 \%$, it appeared later. ${ }^{8}$

The exact risk for malignant degeneration within NS remains unknown ${ }^{1,4}$, but it is thought to vary from $0.13 \%-0.2 \%{ }^{2,6}$. An increased risk seems to exist when the NS is congenital or acquired in infancy, of macular type, and larger than $4 \mathrm{~cm}$ (especially giant and zosteriform variants). ${ }^{2,4,6,8}$ Once a melanoma develops, it has a similar prognosis and management as compared with other melanomas. ${ }^{6}$

There is no standardized management approach for NS. Overall, patients should be advised to perform self-examination to monitor for any changes in their nevi and have a structured longterm follow-up with the help of sequential digital dermatoscopy and, if available, reflectance confocal microscopy. ${ }^{1,4,5,6,9,10}$ This strategy, coupled with judicious use of biopsy for suspicious areas, seems to assist in the early detection of melanoma. ${ }^{1,5,10}$ Prophylactic NS excision without pathologic confirmation of dysplasia or malignancy is not supported by the available literature. ${ }^{1,2}$ In the setting of dysplasia or malignant melanoma, complete NS excision is reasonable, size permitting, in order to eliminate the "faulty background" and to search for any multifocal melanomas. ${ }^{1,2}$

NS is an entity that requires further study, in order to clarify its biologic behavior and allow future development of standardized management guidelines. To the best of our knowledge, this is the fourth report in the literature of synchronous melanomas arising within NS.]

\section{REFERENCES}

1. Meguerditchian AN, Cheney RT, Kane JM 3rd. Nevus Spilus with Synchronous Melanomas: Case Report and Literature Review. J Cutan Med Surg. 2009;13:96101.

2. Corradin MT, Giulioni E, Fiorentino R, Santeufemia DA, Re GL, Vettorello A. In situ malignant melanoma on nevus spilus in an elderly patient. Acta Dermatovenerol Alp Pannonica Adriat. 2014;23:17-9.

3. Manganoni AM, Pavoni L, Farisoglio C, Sereni E, Calzavara-Pinton P. Report of 27 cases of naevus spilus in 2134 patients with melanoma: is naevus spilus a risk marker of cutaneous melanoma? J Eur Acad Dermatol Venereol. 2012;26:129-30.

4. Karam SL, Jackson SM. Malignant Melanoma Arising Within Nevus Spilus. Skinmed. 2012;10:100-2.

5. Piana S, Gelli MC, Grenzi L, Ricci C, Gardini S, Piana S. Multifocal melanoma arising on nevus spilus. Int J Dermatol. 2006;45:1380-1.

6. Corradin MT, Zattra E, Fiorentino R, Alaibac M, Belloni-Fortina A. Nevus Spilus and Melanoma: Case Report and Review of the Literature. J Cutan Med Surg. 2010;14:85-9.

7. Perkinson NG. Melanoma arising in café au lait spot of neurofibromatosis. Am J Surg. 1957;93:1018-20.
8. Abecassis S, Spatz A, Cazeneuve C, Martin-Villepou A, Clerici T, Lacour JP, et al. Melanoma within naevus spilus: 5 cases. Ann Dermatol Venereol. 2006;133:323-8.

9. Tavoloni Braga JC, Gomes E, Macedo MP, Pinto C, Duprat J, Begnami MD, et al. Early detection of melanoma arising within nevus spilus. J Am Acad Dermatol. 2014;70:e31-2.

10. Haenssle HA, Kaune KM, Buhl T, Thoms KM, Padeken M, Emmert S, et al. Melanoma arising in segmental nevus spilus: Detection by sequential digital dermatoscopy. J Am Acad Dermatol. 2009;61:337-41.
MAILING ADDRESS:
Maria Helena Toda Sanches de Brito
Rua Professor Lima Basto, 1099-023
Lisboa, Portugal
E-mail: helenatodabrito@gmail.com

How to cite this article: Brito MHTS, Dionísio CSNM, Fernandes CMBM, Ferreira JCM, Rosa MJMPMC, Garcia MMAPS. Synchronous melanomas arising from nevus spilus. An Bras Dermatol. 2017;92(1):107-9. 